\title{
3D Myocardial Contrast Echocardiography
}

\author{
Katsuomi Iwakura \\ Division of Cardiology, Sakurabashi Watanabe Hospital \\ Japan
}

\section{Introduction}

Early restoration of coronary perfusion is the most important objective in the management of ST-segment elevation myocardial infarction (STEMI), and primary percutaneous coronary intervention (PCI) is established as the most effective strategy for it. Advances in interventional techniques and pharmacological therapy have made it possible to achieve Thrombolysis in Myocardial Infarction (TIMI) grade 3 flow in as many as $95 \%$ of patients undergoing primary PCI. Nevertheless, optimal myocardial perfusion is not achieved in approximately $15 \%$ of patients despite of successful opening of infarct-related artery. The inadequate myocardial perfusion, or "no-reflow" phenomenon, may be caused by microvascular damage after myocardial ischemia, distal coronary emboli resulting from thrombus, platelets and atheroma, in situ thrombosis, vasospasm, or cell necrosis and regional inflammatory responses induced by reperfusion. The no-reflow phenomenon is associated with worse functional and clinical outcomes after STEMI. It was linked to larger infarction size, lower ejection fraction, ventricular arrhythmias(Aiello et al.,1995), early congestive heart failure(Ito et al.,1996), and even cardiac rupture(Morishima et al.,1995). It may have an adverse effect on left ventricular (LV) remodeling(Gerber et al.,2000). Therefore, detection of no-reflow early after primary 'PCI is important for the risk stratification of patients with STEMI. Several invasive and non-invasive imaging modalities have been developed to detect no-reflow. We had focused on one of these modalities, myocardial contrast echocardiography (MCE), and used it in several clinical studies to investigate the pathogenesis of the no-reflow. In this article, we investigated the ability of newly developed, real-time 3D MCE to assess the microvascular dysfunction in patients with AMI.

\section{Imaging modalities for assessment of the no-reflow phenomenon}

No-reflow can be assessed during PCI with Thrombolysis In Myocardial Infarction (TIMI) flow grade(TIMI Study Group,1985), with TIMI-myocardial perfusion grade(van 't Hof et al.,1998) (TMPG), or with coronary flow velocity pattern assessed by Doppler guidewire(Iwakura et al.,1996). It can be better quantified by noninvasive imaging techniques, such as myocardial contrast echocardiography (MCE), cardiac CT, and contrastenhanced cardiac magnetic resonance (CMR).

\subsection{CMR and contrast-enhanced CT}

CMR using gadolinium can diagnose the no-reflow as: 1) lack of gadolinium enhancement during first pass (microvascular obstruction); and 2) lack of gadolinium enhancement within a 
necrotic region, identified by late gadolinium hyperenhancement (Albert et al.,2006) (Ingkanisorn et al.,2004). There is a good correlation between gadolinium enhancement during first pass and TMPG, thus suggesting that these two parameters might reflect the microvascular integrity within the infarct zone (Porto et al.,2007). There is a significant correlation between area of microvascular obstruction and that of hyperenhancement on CMR (Lund et al.,2004). Presence of substantial microvascular obstruction on CMR also predicts LV remodeling (Gerber et al.,2000) (Hombach et al.,2005) and major adverse cardiac events (Hombach et al.,2005). Contrast enhanced multi-detector CT also delineates infarct zone as hyperenhancement area. Transmural hyperenhancement was observed immediately after successful PCI, and its area was correlated with non-viable area assessed by dobutamine stress echocardiography (Habis et al.,2007). Thus, hyperenhancement observed early after PCI on contrast enhanced CT could be associated with the no-reflow phenomenon.

\subsection{Myocardial contrast echocardiography (MCE)}

MCE uses ultrasonic contrast agent containing microbubbles which are strong scatters in an ultrasonic field and send compression and rarefaction waves back to the scanner. MCE has been proven useful in evaluating patients with AMI receiving reperfusion therapy. Ito et al. examined myocardial microvascular perfusion with MCE in patients with AMI, and found that some patients showed a lack of contrast enhancement (no-reflow) after successful PCI(Ito et al.,1992). Their finding is the first clinical report of the no-reflow phenomenon in patients with AMI. They demonstrated patients with substantial no-reflow have poor functional and clinical outcomes after AMI (Ito et al.,1996). They performed MCE using intracoronary injection of fragile microbubbles through catheter. The no-reflow phenomenon was also observed by MCE with intravenous administration of stable microbubbles, which is capable of passing through pulmonary circulation and into coronary microcirculation (Porter et al.,1998). These studies indicated that no-reflow phenomenon is observed in $25-40 \%$ of patients receiving successful primary PCI resulting into TIMI-3 flow grade. The substantial myocardial contrast defect on MCE predicts poor recovery of contractile function (Ito et al.,1992)(Balcells et $a l ., 2003)$ and is associated with both death and recurrent infarction on the later stage (Dwivedi et al.,2007). Multicenter studies have recently demonstrated that the extent of microvascular damage, assessed on day 1 after reperfusion therapy in AMI, is the most powerful independent predictor of development of LV remodeling (Galiuto et al.,2008).

Accumulating study results proved the usefulness of MCE as a clinical tool for evaluating myocardial perfusion, though no contrast agent is approved for this indication currently. MCE has its pros and cons comparing to other imaging modalities such as CMR (Table 1).

\begin{tabular}{lll}
\hline & CMR & MCE \\
\hline Contrast Agent & Gadomium & Microbubbles \\
Resolution & High Spatial Resolution & High Temporal Resolution \\
Image quality & Good in almost all cases & Sometimes inappropriate \\
Reproducibility & Excellent & Moderate \\
Acquisition & About 30 minutes & Real-time \\
Image mode & 2D/3D & 2D \\
Measurement & Area/Volume & Area \\
Operation & In MRI lab & Bedside / in cath-lab \\
\hline
\end{tabular}

Table 1. Characteritics of CMR and MCE 
MCE is a very easy method not requiring a large apparatus, and it can be performed not only in the echo-lab but also in bed side or in the cath-lab. MCE is a suitable imaging modality for assessing myocardial perfusion soon after PCI. On the other hand, interpretation of MCE depends on the quality of obtained images and it could be difficult to obtain reliable images in some cases. Quantification of contrast enhancement on MCE is possible but is still difficult and requires complicated techniques. Even the subjective evaluation of myocardial perfusion is difficult in some cases because of attenuation, lung and rib shadowing, apical bubble distraction by ultrasound.

\subsection{Potential of real-time 3D-MCE}

3D-image reconstruction is the other advantage of CMR over MCE. Precise assessment of myocardial perfusion in a whole LV can be achieved by 3D-imaging. 2D-MCE observes myocardial opacification only on very limited slices, and assessment of contrast defect could be insufficient. Most of myocardial wall thickening is determined by contractile function of the subendocardial layer (Hashimoto et al.,2003), and recovery of contractility after AMI mostly depends on the viability of the subendocardial layer (Garot et al.,2000). Ischemic myocardial injury progress from endocardial- to epicardial layers (wavefront phenomenon), and subendocardium is the most vulnerable layer to ischemic insults (Grattan et al.,1986). Assessment of subendocardial perfusion is important for prediction of contratile function after AMI. 2D-MCE has only limited ability to visualize subendocardial perfusion.

3D- echocardiography visualizes the whole LV, and it is superior to 2D-echocardiography in assessment of regional wall motion abnormalities (Corsi et al.,2005). It also visualizes endocardial surface structure within a beating heart (Inoue et al.,2006). In the next chapter, we investigated whether real-time 3D-MCE could assess subendocardial perfusion in patients with AMI undergoing primary $\mathrm{PCI}$, and compared its perfusion patterns to those obtained with 2D-MCE (Iwakura et al.,2007). We also compared the ability of these two modalities to assess infarct size and to predict functional recovery.

\section{Assessment of subendocardial perfusion by real-time 3D-MCE}

\subsection{Study population and protocol}

Between October 2004 and December 2005, consecutive 68 patients with AMI underwent primary PCI within 24 hours after symptom onset, and subsequently underwent intracoronary 2D- and 3D-MCE study. The diagnosis of AMI was based on the chest pain prolonged $\geq 30$ minutes, ST segment elevation of $\geq 2 \mathrm{~mm}$ in at least two contiguous electrocardiograph leads, and greater than 3 fold increase in serum creatine kinase (CK) levels. Seven patients were excluded because of poor echocardiograph images, including 2 patients in whom 2D-MCE was adequate but 3D-MCE was suboptimal. We excluded 14 patients who did not undergo follow-up left ventriculography (LVG) study. Therefore, the final study population consisted of 47 patients.

After the admission, we performed echocardiography examination with SONOS 7500 (Philips Medical Systems), and defined the risk area as myocardial segments showing dyskinesia, akinesia or severe hypokinesia. After aspirin $(243 \mathrm{mg})$ and intravenous heparin 
(100 U/kg) administration, we performed coronary angiography (CAG) using the right femoral approach. We determined the culprit lesion and performed primary PCI to achieve the residual diameter stenosis $<25 \%$. After the PCI procedure, we assessed TMPG on CAG from the view chosen to minimize superimposition of non-infarcted territories(Gibson et al.,2000).

A mean of 15 minutes after the last PCI procedure, we performed 2D-MCE with SONOS 7500 using a S4 transducer. We made microbubbles of a mean size of $12 \mu \mathrm{m}$ by sonicating iodinated contrast medium, Ioxagate (Hexabrix-320, Tanabe), using an ultrasonic homogenizer with a sterilized tip (Figure 1). We injected $2 \mathrm{~mL}$ of sonicated medium into the right coronary artery in patients with inferior wall AMI and into the left coronary artery in those with anterior or posterior wall AMI. We recorded 2D-echocardiogram from the apical two- or four-chamber view. Then, we performed real time 3D-MCE using Live 3D system without ECG gating. We observed 3D images from apical 2- or 4-chamber view with an X4 matrix array transducer on second harmonic mode. We adjusted time-gain compensation and lateral gain control carefully to obtain clear view of endocardial surface. Then, we injected microbubbles into culprit coronary artery again, and recorded a 3D-MCE image for 20 heartbeats. All MCE images were digitally stored for the further analysis.

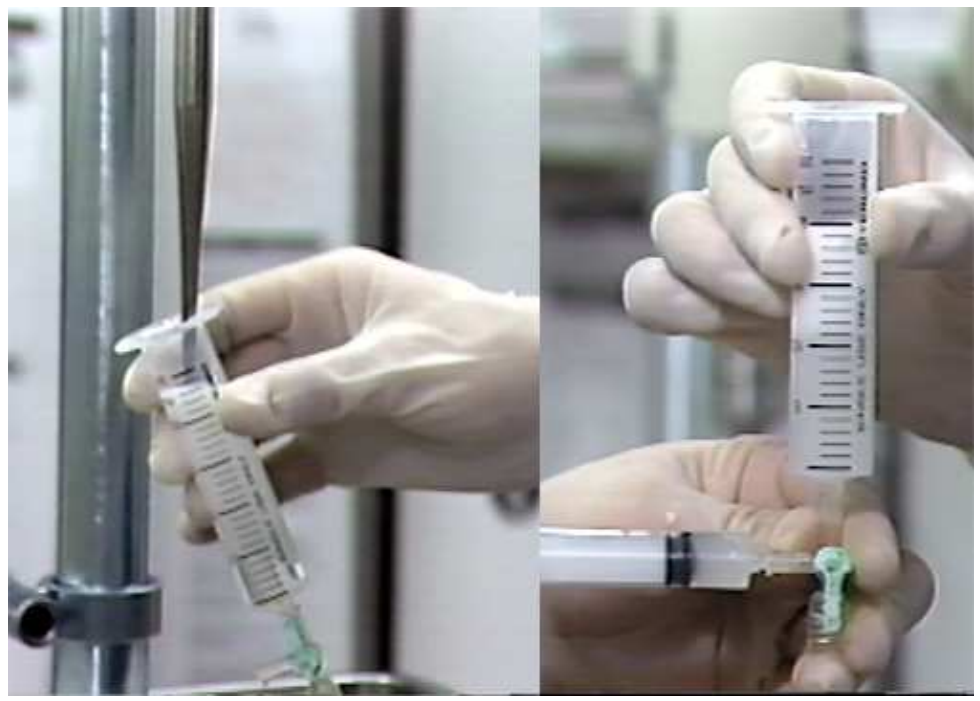

Fig. 1. Sonification of contrast medium to make microbubbles for MCE

We performed LVG in 42 patients $(89.4 \%)$ following MCE study, and measured left ventricular end-diastolic and end-systolic volume index (LVEDVI and LVESVI, $\mathrm{mL} / \mathrm{m}^{2}$ ) and LV ejection fraction (LVEF, \%) by the biapical Simpson's rule. Regional wall motion (RWM, $\mathrm{SD} /$ chord) within the culprit artery territory was analyzed using the centerline method. Follow-up coronary angiography and LVG were performed in all patients at a mean of $4.6 \pm 2.7$ months later.

An experienced echocardiographer analyzed 2D-MCE images to determine myocardial perfusion within the risk area. We used the apical long-axis view or apical 4-chamber view 
for the patients with anterior wall AMI and posterior wall AMI and the apical 2-chamber view for the patients with inferior wall AMI. We divided LV wall into myocardial segments based on 16-segment model endorsed by American Society of Echocardiography(Cerqueira et al.,2002), and scored myocardial opacification in each segment as 1 (homogenous opacification), 0.5 (patchy opacification or opacification only in epicardium), or 0 (no opacification)(Ragosta et al.,1994). We calculated the averaged contrast score by dividing the sum of contrast scores in the segments within risk area by the number of these segments. We graded myocardial perfusion in each patient as good- (the averaged score $=1)$, poor- $(0.5 \leq$ score $<1.0$ ) and no-reflow (score $<0.5$ ).

A sonographer blinded to 2D-MCE data assessed subendocardial contrast opacification on end-systolic 3D-MCE images. For assessing the risk area and myocardial perfusion, we used the echo windows to include the whole risk area within 3D echocardiograph image. We observed myocardial opacification from LV cavity side. The "shade" mode was activated to reduce the effect of opacification from the epicardial layers. If necessary, some parts of the LV were cropped to obtain clear image of endocardial surface (Figure 2). Risk area was defined as myocardium showing no contraction of endocardial surface. We divided whole LV into myocardial segments corresponding to those of 16-segment model in 2Dechocardiogram(Kapetanakis et al.,2005), and scored endocardial opacification within each segment as 1 (enhancement as good as that within nearby normal segment), 0.5 (enhancement was observed but not as strong as that within the normal area), or 0 (no clear enhancement). We calculated the averaged endocardial contrast score in the segments within the risk area. We graded myocardial opacification in endocardium (MOE) into 3 groups; good- (the averaged score $=1)$, poor- $(0.5 \leq$ score $<1.0)$ and no-MOE (score $<0.5)$.
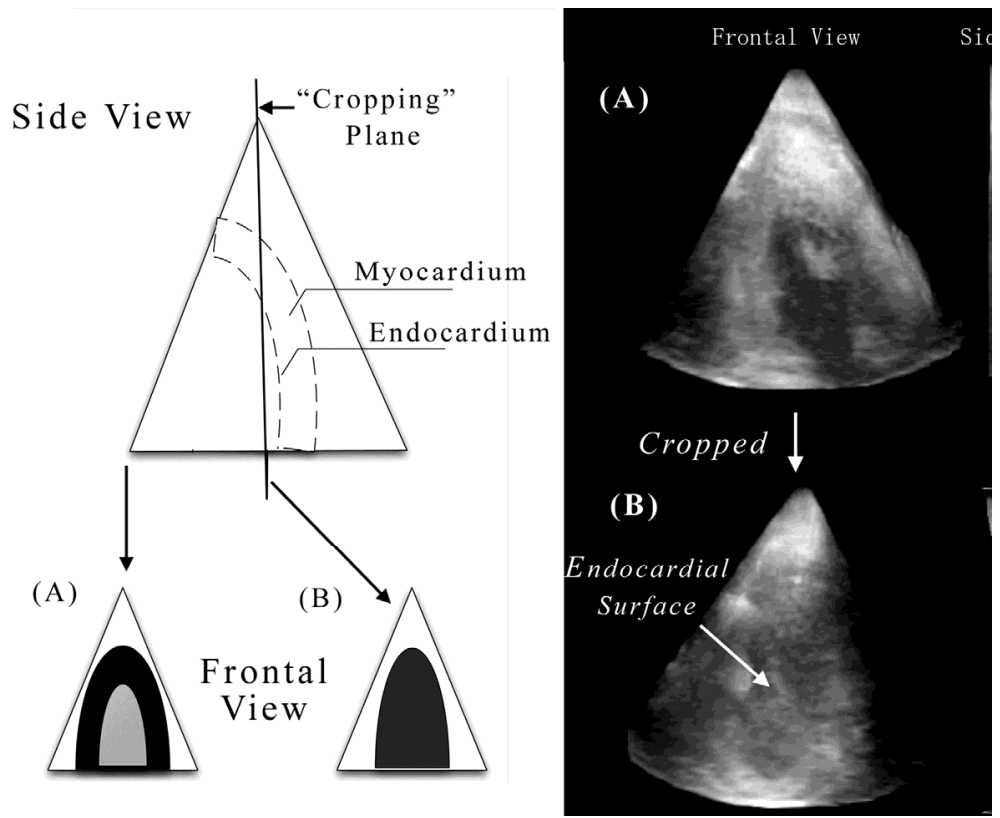

Fig. 2. Cropping of 3D-MCE images to reveal endocardial surface 
All data are expressed as mean \pm SD. We made comparisons by one-way ANOVA for continuous variables, and significance of difference was calculated with Tukey's HSD test for factor analysis. Categorical variables were compared with the Fisher's exact test. The differences in the changes of RWM from the initial- to the follow-up LVG study among the groups were analyzed with multivariate analysis of variance (MANOVA). Differences were considered significant at $P<0.05$ (two-sided).

\subsection{Patient characteristics}

The mean age of the 47 study patients was $61 \pm 11$ years (range 40 to 81 years), and 38 patients $(80.9 \%)$ were male. The culprit artery was the left anterior descending artery in 28 patients, the left circumflex artery in 6 patients and the right coronary artery in 13 patients. Multivessel disease was observed in 12 patients. The mean time from the symptom onset to coronary reperfusion was $12.3 \pm 14.2$ hours. The peak CK and CK-MB level was $3069 \pm 2490$ $\mathrm{IU} / \mathrm{L}$ and $235 \pm 185 \mathrm{IU} / \mathrm{L}$, respectively.

\subsection{Representative cases of 3D-MCE}

Figures 3 shows 2D- and 3D-MCE images in a patient with inferior wall AMI. 2D-MCE (upper left) showed good-reflow within the risk area (between arrows). On 3Dechocardiograph before injection, endocardial surface of area at risk was revealed through cropping (lower left). After microbubble injection, contrast enhancement was observed within the risk area, and we judged this case as good-MOE. Peak CK and CK-MB of this case were $1977 \mathrm{IU} / \mathrm{L}$ and $118 \mathrm{IU} / \mathrm{L}$, respectively.

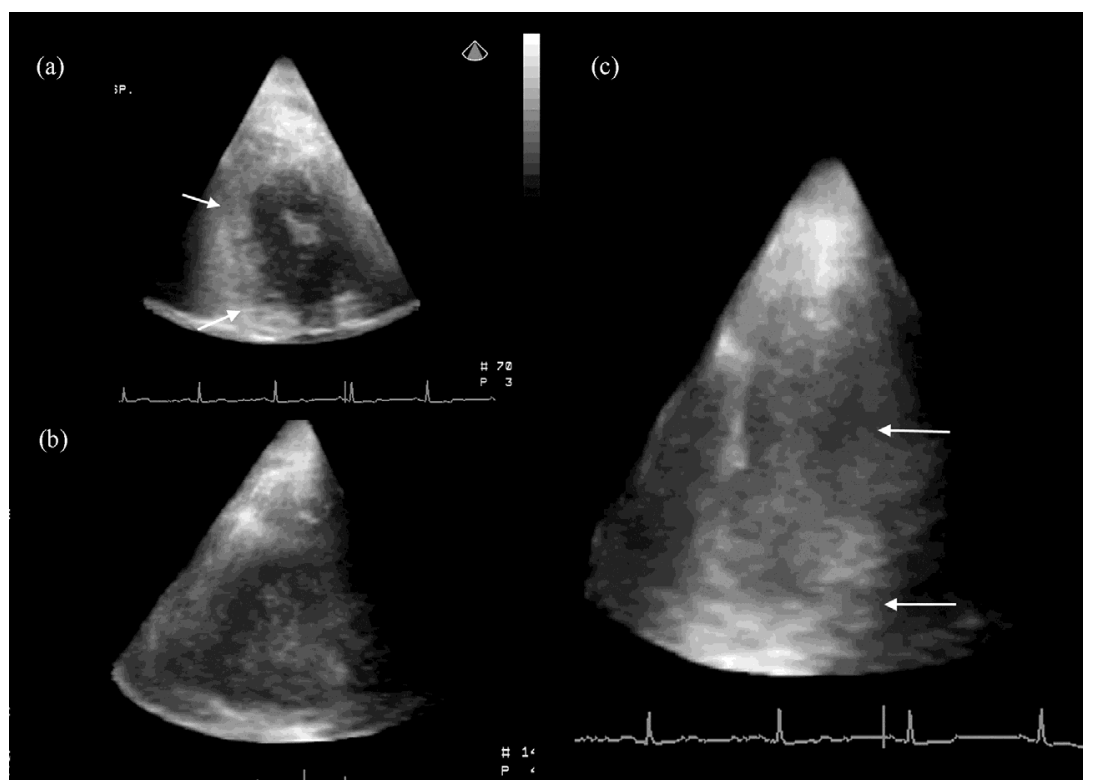

Fig. 3. A representative case showing good-reflow on 2D-MCE (a) and good-MOE on 3DMCE (c). 
Figure 4 showed MCE images in a patient with posterior wall AMI. 2D-MCE (upper left) showed good-reflow within the risk area (between arrows). 3D-MCE (right) showed almost no endocardial contrast enhancement (no-MOE) within the risk area (between arrows). A good contrast enhancement was observed in the normal myocardium around the risk are (Compare it to the 3D-image before contrast injection, on lower left). Peak CK and CK-MB of this case were $4758 \mathrm{IU} / \mathrm{L}$ and $414 \mathrm{IU} / \mathrm{L}$, respectively.

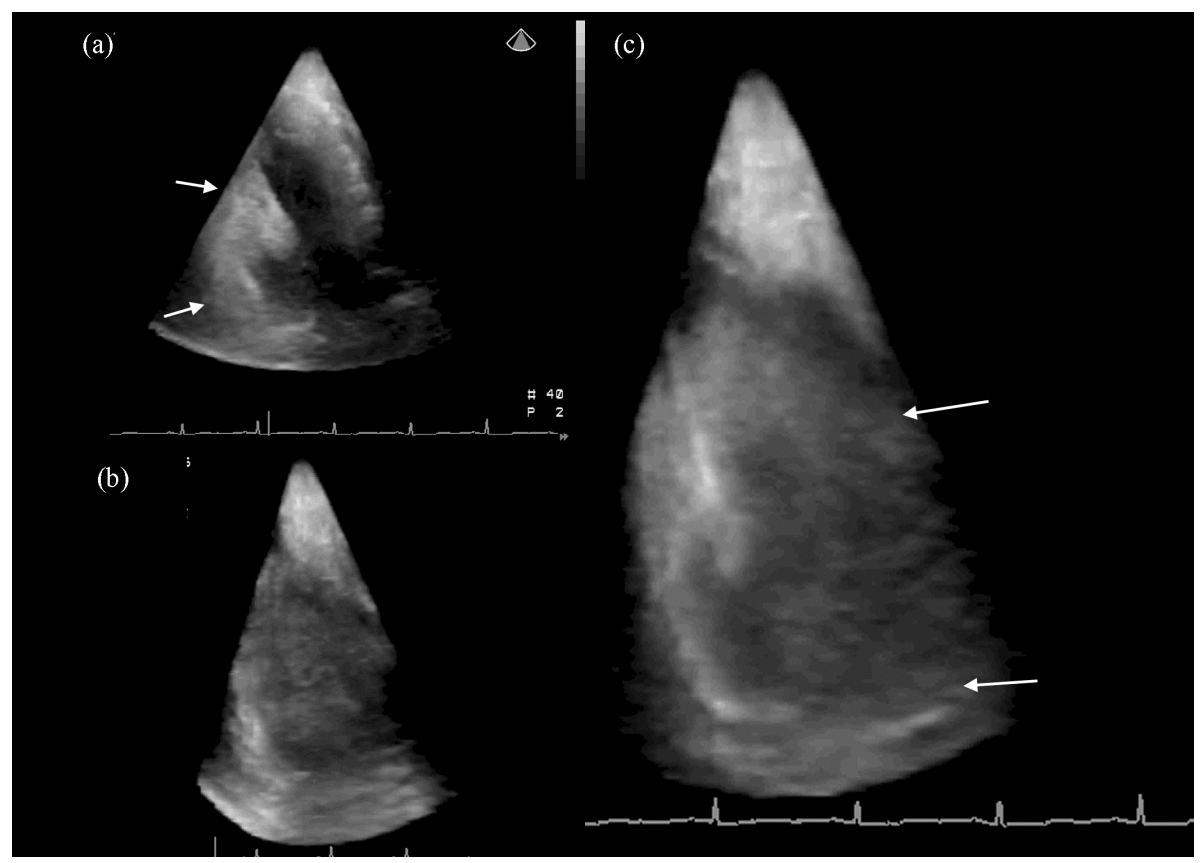

Fig. 4. A case with posterior AMI showing good-reflow on 2D-MCE (a) but no-MOE on 3D$\operatorname{MCE}(\mathrm{c})$.

Figure 5 showed interesting images obtained in other case with anterior wall AMI. Good opacification was observed on endocardial surface of septum on 3D-MCE at 790msec after injection of microbubbles (b). Then, a jet of contrast medium was observed to flow out directly from the apical myocardium (arrow) at $2220 \mathrm{msec}$ (c) and $2990 \mathrm{msec}$ (d) after injection ( (e) and (f) are zoomed images of (c) and (d)). These images demonstrated that endocardial hemorrhage occurred immediately after AMI. Possibly rupture of subendocardial hematoma occurred in this case (Iwakura, 2011). 


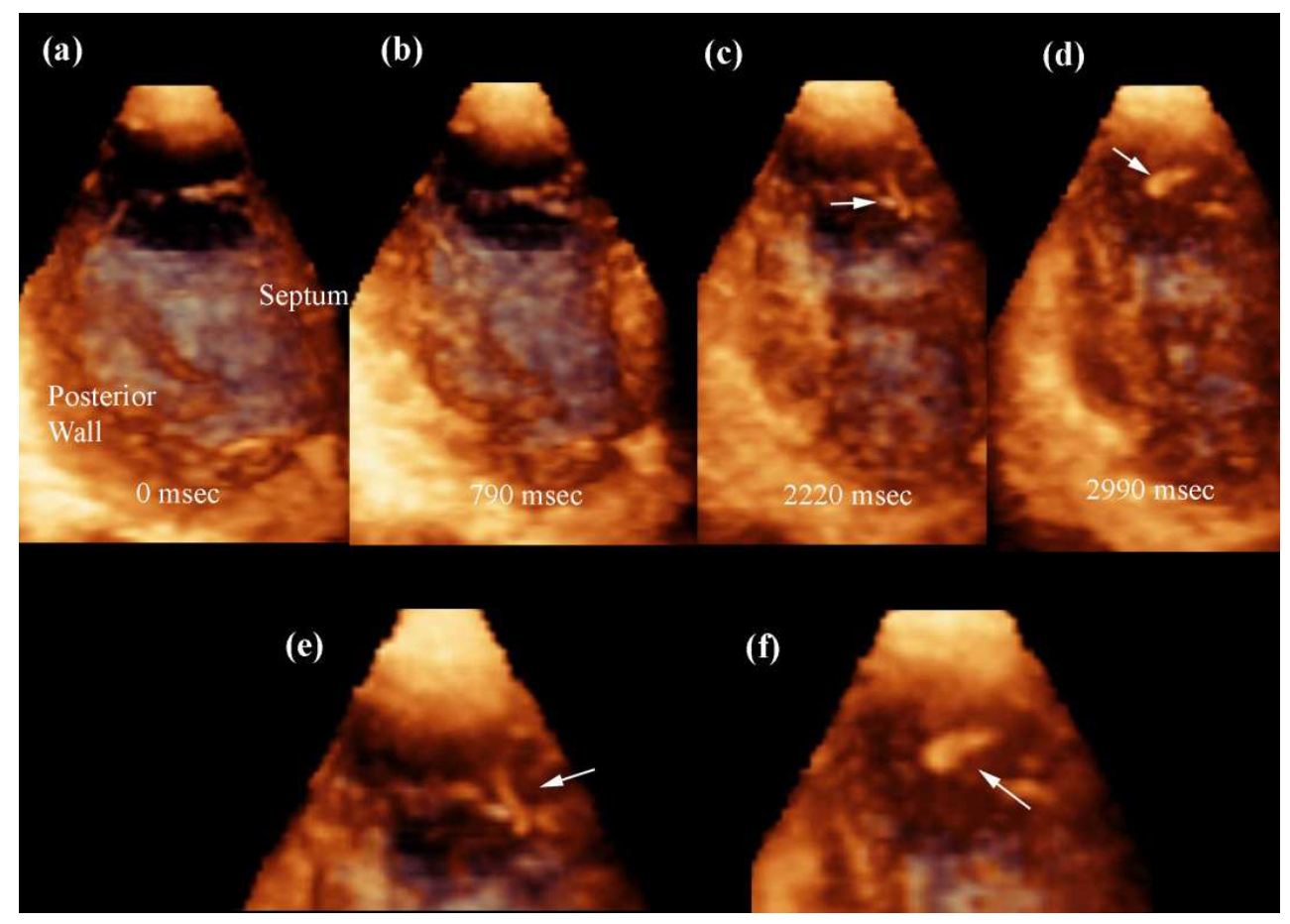

Fig. 5. Endocardial hemorrhage observed immediately after primary PCI.

\subsection{Assessment of endocardial perfusion with 3D-MCE.}

2D-MCE showed good-reflow in 31 out of 47 study patients $(66.0 \%)$, poor-reflow in 9 patients $(19.1 \%)$ and no-reflow in 7 patients $(14.9 \%)$. Patient characteristics of each group were illustrated in Table 2. The incidence of anterior wall AMI was significantly higher in the no- and poor-reflow than the good-reflow $(p=.01)$. The additional ST elevation after reperfusion was more frequently observed in the no-reflow $(p=.04)$. There were significant differences in the incidence of the multivessel disease $(p=.02)$ and the collateral grade $(p=.03)$ among three subsets (Table 2). 


\begin{tabular}{|c|c|c|c|c|c|c|c|c|}
\hline & \multicolumn{4}{|c|}{ 2D-MCE } & \multicolumn{4}{|c|}{ 3D-MCE } \\
\hline & Good-reflow & Poor-reflow & No-reflow & $\mathbf{P}$ & Good-MO & Poor-MOE & No-MOE & $\mathbf{P}$ \\
\hline Number of Patients & 31 & 9 & 7 & & 17 & 16 & 14 & \\
\hline Age, $y$ & $62 \pm 11$ & $60 \pm 10$ & $55 \pm 11$ & .30 & $63 \pm 12$ & $63 \pm 9$ & $55 \pm 10$ & .08 \\
\hline Gender, male/female & $\mathbf{2 4} / 7$ & $9 / 0$ & $5 / 2$ & .25 & $15 / 2$ & $13 / 3$ & $10 / 4$ & .50 \\
\hline Peak CK, IU/L & $2516 \pm 2186$ & $3195 \pm 2209$ & $5240 \pm 3040$ & .03 & $1166 \pm 1081$ & $2796 \pm 1700$ & $5583 \pm 2345$ & $<.0001$ \\
\hline Peak CK-MB, IU/L & $204 \pm 185$ & $259 \pm 166$ & $\mathbf{3 4 9} \pm \mathbf{1 7 7}$ & .16 & $100 \pm 100$ & $261 \pm 189$ & $383 \pm 144$ & $<.0001$ \\
\hline Diabetes Mellitus, n ( $\%)$ & $12(38.8)$ & $4(44.4)$ & 3 (42.9) & .94 & $6(35.3)$ & 7 (43.8) & $6(42.9)$ & .86 \\
\hline Hypertension, $\mathbf{n}(\%)$ & $19(61.3)$ & $5(55.6)$ & 5 (71.4) & .81 & $10(58.8)$ & $11(68.8)$ & 8 (57.1) & .77 \\
\hline Hyperlipidemia, n ( $\%)$ & 16 (51.6) & $6(66.7)$ & $2(28.6)$ & .32 & 12 (70.6) & 5 (31.3) & $7(50.0)$ & .08 \\
\hline Smoking, n ( $\%)$ & 22 (71.0) & 8 (88.9) & $6(85.7)$ & .44 & 12 (70.6) & $12(75.0)$ & $12(85.7)$ & .60 \\
\hline Onset to reperfusion, $h$ & $13.0 \pm 13.1$ & $15.1 \pm 20.9$ & $5.9 \pm 4.9$ & .41 & $11.2 \pm 13.4$ & $18.7 \pm 17.9$ & $5.8 \pm 4.3$ & .04 \\
\hline Stent implantation, $\mathrm{n}(\%)$ & 29 (93.4) & $9(100)$ & 5 (71.4) & .10 & $16(94.1)$ & $15(93.8)$ & $12(85.7)$ & .65 \\
\hline Thrombectomy, n ( $\%)$ & $21(67.7)$ & 7 (77.8) & $6(85.7)$ & .58 & $12(70.6)$ & $11(68.8)$ & $11(78.6)$ & .82 \\
\hline Anterior wall MI, n (\%) & $17(54.8)$ & $6(66.7)$ & 5 (71.4) & .64 & $11(64.7)$ & 7 (43.8) & 10 (71.4) & .26 \\
\hline Multivessel disease, $n$ & 8 (25.8) & $3(33.3)$ & 1 (14.3) & .68 & $4(23.5)$ & 5 (31.2) & 3 (21.4) & .80 \\
\hline ST re-elevation, $n(\%)$ & $11(35.5)$ & 1 (11.1) & $5(71.4)$ & .04 & $2(11.8)$ & $7(\mathbf{4 3 . 8})$ & $8(57.1)$ & .02 \\
\hline
\end{tabular}

Table 2. Clinical parameters of the study patients.

Good-MOE was observed on 3D-MCE only in 17 patients (36.2\%). Poor-MOE was observed in 16 patients $(34.0 \%)$ and no-MOE in 14 patients $(29.8 \%)$. Among the 31 patients with goodreflow on 2D-MCE, only 14 patients (45.2\%) showed good-MOE, and 4 patients $(12.9 \%)$ showed no-MOE. In contrast, all 7 patients with no-reflow on 2D-MCE showed no-MOE on 3D-MCE (Table 3).

\begin{tabular}{lcccc}
\hline & \multicolumn{3}{c}{ 3D-MCE } & \\
\cline { 2 - 4 } 2D-MCE & Good-MOE, $\mathrm{n}$ & Poor-MOE, $\mathrm{n}$ & No-MOE, $\mathrm{n}$ & Total, $\mathrm{n}$ \\
\hline Good-reflow, $\mathrm{n}$ & 14 & 13 & 3 & 31 \\
Poor-reflow, $\mathrm{n}$ & 3 & 3 & 3 & 9 \\
No-reflow, $\mathrm{n}$ & 0 & 0 & 7 & 7 \\
\hline Total, $\mathrm{n}$ & 17 & 16 & 14 & 47 \\
\hline
\end{tabular}

Table 3. Distribution of myocardial perfusion grade on 2D- and 3D-MCE

Among the 31 patients with good-reflow, only 19 patients (61.3\%) showed TMPG-3, and 6 patients showed TMPG-0/1 on CAG after PCI. On the other hand, 16 out of 17 patients (94.1\%) with good-MOE showed TMPG-3. 


\subsection{Prediction of infarct size with 2D- and 3D-MCE}

Among the 3 groups classified with 2D-MCE, the no-reflow had the highest peak-CK value (5240 $\pm 3040 \mathrm{IU} / \mathrm{L})$, followed by the poor-reflow $(3195 \pm 2209 \mathrm{IU} / \mathrm{L})$ and the good-reflow $(2516 \pm 2186 \mathrm{IU} / \mathrm{L})$. The differences in peak CK among three subsets were significant $(p=.03)$, and the no-reflow had significantly higher peak CK than the good-reflow. However, the differences in peak CK between the poor-reflow and the good- or the no-reflow did not reach statistical significance (Figure 6). There were no significant differences in CK-MB values among the three subsets $(\mathrm{p}=0.16)$.
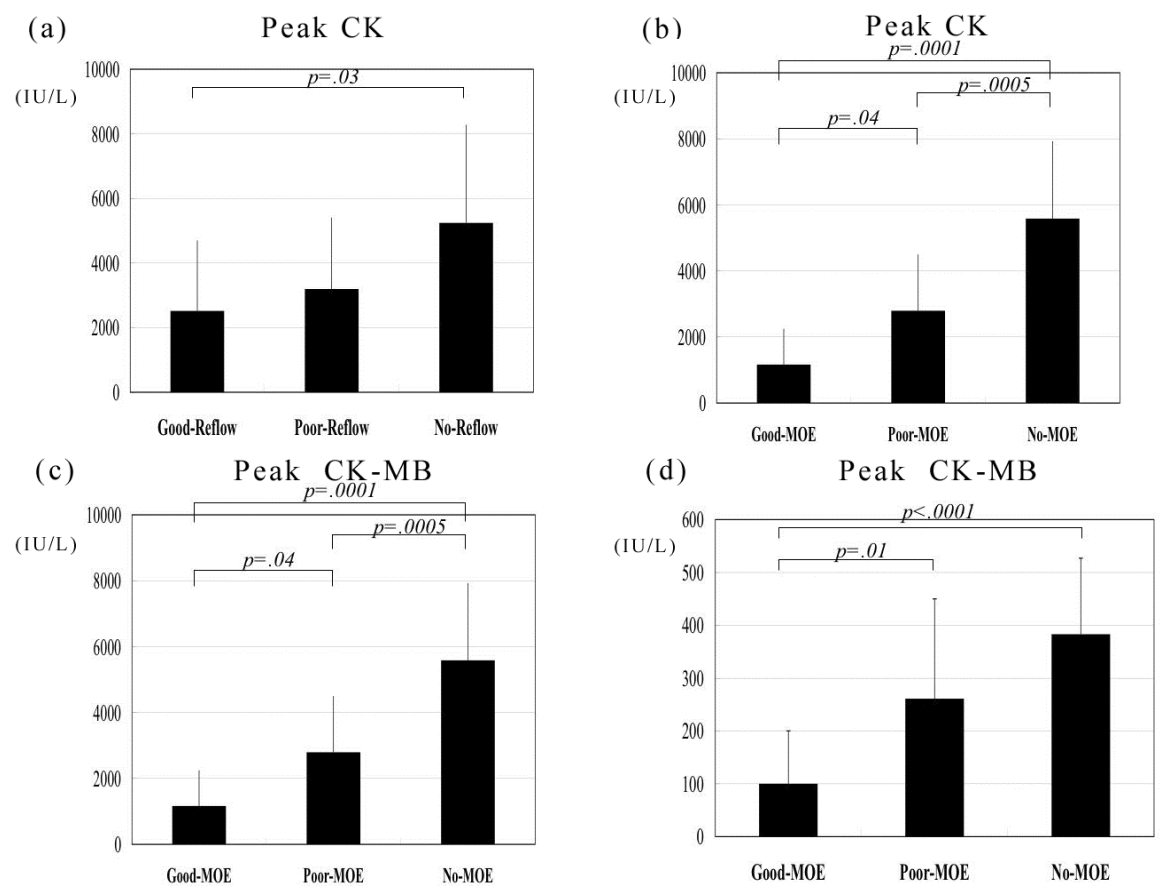

Fig. 6. Peak CK/CK-MB and myocardial perfusion grade on 2D/3D-MCE

The no-MOE on 3D-MCE also had the highest peak CK value ( $5583 \pm 2345 \mathrm{IU} / \mathrm{L})$, followed by the poor-MOE $(2796 \pm 1700 \mathrm{IU} / \mathrm{L})$ and by the good $-\mathrm{MOE}(1166 \pm 1080 \mathrm{IU} / \mathrm{L})$. The differences in peak CK were significant not only among 3 groups $(\mathrm{p}<.0001)$ but also in each pair of groups (Figure 6). Moreover, there were significant differences in CK-MB among 3 groups $(p<.0001)$. The good-MOE had significantly lower CK-MB then the poor- $(p=.01)$ or the noMOE $(p<.0001)$ (Figure 6). These results indicated that myocardial perfusion grade by 3DMCE predicted infarct size more distinctively than that by 2D-MCE does.

\subsection{Prediction of LV functional and morphological outcomes with 2D- and 3D-MCE}

At baseline study, there was no significant difference in RWM among 3 groups based on 2D-MCE. The good-MOE had the highest RWM among 3 groups based on 3D-MCE at 
baseline study (good-/poor-/no-MOE $=-2.28 \pm 0.91 /-3.03 \pm 0.67 /-2.99 \pm 0.71, p=0.02$ ). A mean of $4.6 \pm 2.7$ months later, RWM of the no-reflow $(-2.84 \pm 0.83)$ was lower than that of the good-reflow $(-1.37 \pm 1.11, p=.004)$. However, RWM was not statistically different between the poor-reflow (-1.73 \pm 0.71$)$ and the good-reflow or the no-reflow (Figure 7$)$. On the other hand, there were significant differences in RWM in each pair of the 3 groups defined by 3D-MCE (Figure 7).

(a)

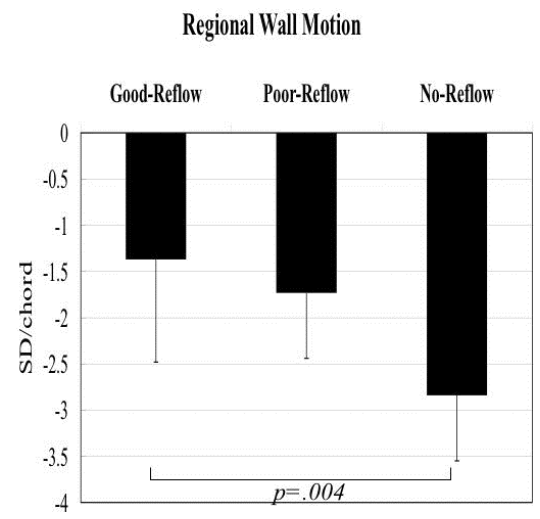

(b)

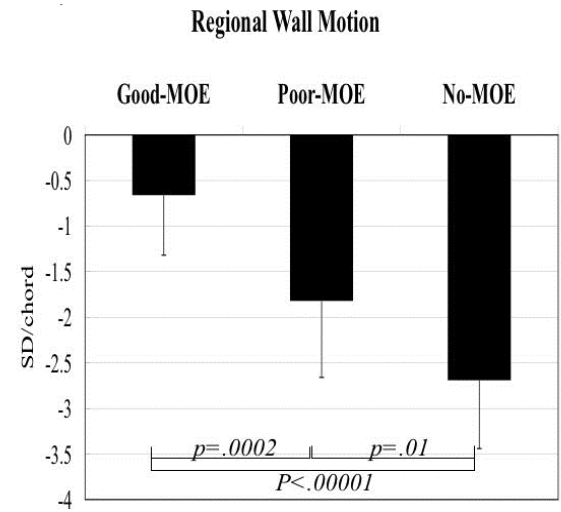

Fig. 7. RWM on the chronic stage and myocardial perfusion grade on 2D/3D-MCE

Among the 42 patients in whom initial ventriculography study was performed, the improvement of RWM from baseline to follow-up study was the highest in the good-MOE, followed by the poor-MOE and the no-MOE $(1.59 \pm 0.98$ vs. $1.19 \pm 0.71$ vs. $0.31 \pm 0.84, p=.001)$. Although RWM improvement was also the highest in the good-reflow, but it showed significant overlap among 3 groups, and no significant differences were observed among them $(p=0.10)$. These results implied that myocardial perfusion pattern assessed with 3DMCE predicts the functional recovery more definitively than that with 2D-MCE.

There were no differences in LVEDVI and LVESVI at baseline study among 3 groups based on 2D-MCE, and there were no significant differences in LVEDVI and LVESVI among 3 groups at 
follow-up study. The no-reflow had significantly lower ejection fraction than the good-reflow at baseline study. At the follow-up study, the good-reflow showed higher LVEF than the noreflow, but LVEF of the poor-reflow showed significant overlap with those of other two subsets (Figure 8). At baseline, LVEDVI was comparable among 3 groups defined by 3D-MCE. The good-MOE had smaller LVESVI than the no-MOE and better LVEF than other two groups on initial LVG. At the follow-up study, the no-MOE had larger LVEDVI than the good-MOE, and larger LVESVI and LVEF fraction than two other groups (Figure 8).

(a)

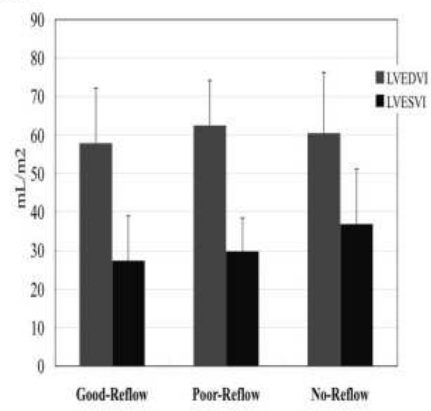

(c)

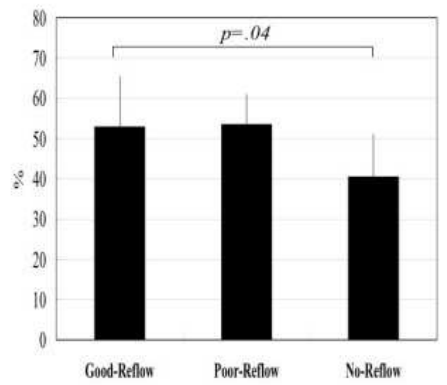

(b)

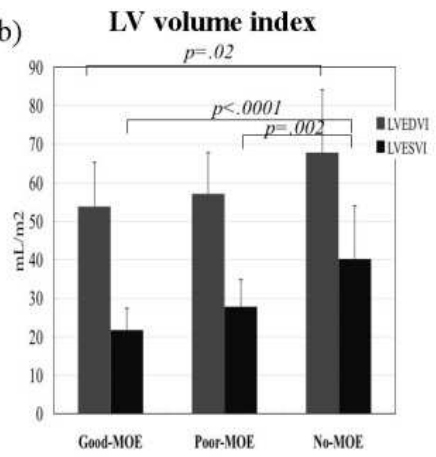

(d)

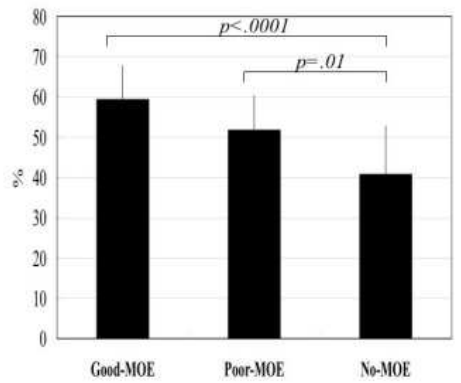

Fig. 8. LV volume index and LVEF on the chronic stage

\section{3D-MCE as a novel method for assessment of subendocardial perfusion}

The present study demonstrated that subendocardial perfusion after coronary recanalization was well visualized with 3D-MCE in patients with AMI. Assessment of subendocardial perfusion with 3D-MCE predicted infarct size and discriminated patients with poor functional outcome more precisely than 2D-MCE. We used intracoronary injection of microbubbles for 3D-MCE rather than intravenous injection because intravenous injection of contrast agent would make LV filled with microbubbles, which make endocardial surface invisible.

\subsection{Comparison between 2D- and 3D-MCE for detection of the no-reflow}

While the no-reflow assessed by 2D-MCE is associated with poor functional recovery after AMI, the microvascular integrity does not always go along with functional recovery on the 
chronic stage(Galiuto et al.,1998). TMPG-3 on coronary angiogram is generally achieved only in less than $30 \%$ of the patients with AMI after primary PCI(Gibson et al.,2000)(Costantini et al.,2004), while good reflow is observed on 2D-MCE in about 70\% of the patients(Ito et al.,1992)(Galiuto et al.,1998)(Porter et al.,1998). TMPG grade was far better correlated with MOE on 3D-MCE than with perfusion pattern on 2D-MCE in the present study. Thus, 2DMCE might overestimate myocardial reperfusion and predict functional recovery excessively(Bolognese et al.,1996).

Myocardial perfusion patterns assessed by 3D-MCE were not necessarily coincident with those with 2D-MCE. The frequency of good-MOE on 3D-MCE was significantly lower than that of good reflow on 2D-MCE (36.2\% vs. $66.0 \%)$ in the present study. Although 2D-MCE might detect subendocardial perfusion defect in some cases(Ragosta et al.,1994), it basically could not well assess transmural differences in myocardial perfusion(Kaul et al.,1992). 3DMCE observes contrast opacification on the endocardial surface directly from the view point of LV cavity, and could assess subendocardial perfusion more precisely than 2D-MCE. Nevertheless, direct observation of subendocardial opacification has some limitations for precise subendocardial perfusion. Contrast signal from the mid-layers might contaminate the subendocardial opacification, even with adjustment of recording conditions to reduce such interference. We used sonicated contrast medium for intracoronary MCE, which contains various sized microbubbles(Ito et al.,1992). Large bubbles work as scatters rather than reflectors of ultrasound, and ultrasound scatter would produce false ultrasound signals in the neighboring myocardium. This interference has the potential for inhibiting the discrimination between endocardium and epicardium on 3D-MCE(Kaul et al.,1992).

While 2D-MCE observed myocardial perfusion only in a slice of the risk area, 3D-MCE observed spatial distribution of perfusion more widely. If the echo-plane on 2D-MCE is not placed at the center area of the infarct zone, we would observe myocardial perfusion at the marginal zone. Microvascular injury in the peripheral zone is not as severe as in the center area, and myocardial perfusion could be overestimated. The 3D-MCE image in Figure 4 showed high contrast enhancement area around the central risk area. This could be hyperemic response occurring at the marginal area. Presence of hyperemia might make 2DMCE overestimate myocardial perfusion.

\subsection{Limitation of the study}

In the present study, we used real-time 3D echocardiography (Live-3D) instead of fullvolume imaging. Real-time $3 \mathrm{D}$ acquisition is limited to an angle of about $30^{\circ}-50^{\circ}$ degrees, and provides only a partial view of LV. To cover the complete LV, acquisition of wide-angle ('full-volume') volumetric data sets using ECG gating is required. 3D-contrast echocardiography, using intravenous contrast agents, assesses LV volumes more precisely than 2D-contrast echocardiography or 3D-echocardiography in patients with a history of myocardial infarction(Jenkins et al.,2009). LV volumes measured with 3D-contrast echocardiography is compatible with those measured on CMR. However, 3D-contrast echocardiography was used to delineate endocardial border clearly (LV opacification), and myocardial perfusion was not assessed in these studies. Full-volume 3D-MCE will not only assess myocardial perfusion within a complete LV, but will be able to measure myocardial volume showing microvascular dysfunction. At the time of the present study, the imageanalysis software for SONOS 7500 was not suitable for observation of contrast enhancement 
in a full-volume image, let alone measurement of the volume of microvascular dysfunction. New software should be developed to analyze a full-volume 3D-MCE image quantitatively.

Despite of the limitation described above, real-time 3D-MCE has some advantages over fullvolume imaging, including direct and easy observation of changes in endocardial structures. In one of the present study patients, we observed eruption of contrast medium from endocardial surface immediately after intracoronary injection of microbubbles. We considered this phenomenon as indicating rupture of subendocardial hematoma. In an experimental model, intramural hemorrhage is not observed early after coronary reperfusion on intracoronary 2D-MCE (Shishido et al.,1997). The contrast defect spread significantly with time after reperfusion in the cases developing intramural hemorrhage, but enhancement immediately after reperfusion was compatible between those with and without hemorrhage (Shishido et al.,1997). 2D-MCE might fail to detect small hemorrhage limited to the subendocardial layer, while 3D-MCE could detect it by direct observation of endocardial surface. Thus, real time 3D-MCE is a promising method not only to observe on endocardial perfusion but also to observe pathological events occurring endocardial surface.

The present real time 3D-MCE technique has other limitations. The spatial resolution of 3Dechocardiograhy is still inferior to that of 2D-echocardiography, which might lead to the poorer 3D-MCE image quality. We evaluated perfusion pattern and MOE only visually, because the present system does not have the objective method to measure myocardial opacification on 3D-images. The quantitative measures using replenishment curves which is available in 2D-MCE (Wei et al.,1998) still could not be performed in 3D-MCE. We hope that the technical progress would soon resolve these technical issues.

\section{Conclusion}

Newly developed, real time 3D-MCE was a feasible method to assess endocardial perfusion in patients with AMI. 3D-MCE assessed infarct size and predicted functional outcomes after AMI better than 2D-MCE did. It also observed endocardial hemorrhage occurred after PCI in one of the study patients. Thus, 3D-MCE is a promising method for assessment of microvascular function and of endocardial structural changes immediately after primary PCI for AMI.

\section{References}

Aiello, E.A., Jabr, R.I. \& Cole, W.C. (1995) Arrhythmia and delayed recovery of cardiac action potential during reperfusion after ischemia. role of oxygen radical-induced no-reflow phenomenon. Circ. Res., Vol.77, No.1, (Jul, 1995), pp. 153-162, ISBN00097330

Ito, H., Maruyama, A., Iwakura, K., Takiuchi, S., Masuyama, T., Hori, M., Higashino, Y., Fujii, K. \& Minamino, T. (1996) Clinical implications of the 'no reflow' phenomenon. a predictor of complications and left ventricular remodeling in reperfused anterior wall myocardial infarction. Circulation, Vol.93, No.2, (Jan, 1996), pp. 223-228, ISBN0009-7322

Morishima, I., Sone, T., Mokuno, S., Taga, S., Shimauchi, A., Oki, Y., Kondo, J., Tsuboi, H. \& Sassa, H. (1995) Clinical significance of no-reflow phenomenon observed on angiography after successful treatment of acute myocardial infarction with 
percutaneous transluminal coronary angioplasty. Am. Heart J., Vol.130, No.2, (Aug, 1995), pp. 239-243, ISBN0002-8703

Gerber, B.L., Rochitte, C.E., Melin, J.A., McVeigh, E.R., Bluemke, D.A., Wu, K.C., Becker, L.C. \& Lima, J.A. (2000) Microvascular obstruction and left ventricular remodeling early after acute myocardial infarction. Circulation, Vol.101, No.23, (Jun, 2000), pp. 2734-2741, ISBN1524-4539

TIMI Study Group (1985) The thrombolysis in myocardial infarction (TIMI) trial. phase i findings.. N. Engl. J. Med., Vol.312, No.14, (Apr, 1985), pp. 932-936, ISBN0028-4793

van 't Hof, A.W., Liem, A., Suryapranata, H., Hoorntje, J.C., de Boer, M.J. \& Zijlstra, F. (1998) Angiographic assessment of myocardial reperfusion in patients treated with primary angioplasty for acute myocardial infarction: myocardial blush grade. zwolle myocardial infarction study group. Circulation, Vol.97, No.23, (Jun, 1998), pp. 2302-2306, ISBN0009-7322

Iwakura, K., Ito, H., Takiuchi, S., Taniyama, Y., Nakatsuchi, Y., Negoro, S., Higashino, Y., Okamura, A., Masuyama, T., Hori, M., Fujii, K. \& Minamino, T. (1996) Alternation in the coronary blood flow velocity pattern in patients with no reflow and reperfused acute myocardial infarction. Circulation, Vol.94, No.6, (Sep, 1996), pp. 1269-1275, ISBN0009-7322

Albert, T.S.E., Kim, R.J. \& Judd, R.M. (2006) Assessment of no-reflow regions using cardiac mri. Basic Res. Cardiol., Vol.101, No.5, (Sep, 2006), pp. 383-390, ISBN0300-8428

Ingkanisorn, W.P., Rhoads, K.L., Aletras, A.H., Kellman, P. \& Arai, A.E. (2004) Gadolinium delayed enhancement cardiovascular magnetic resonance correlates with clinical measures of myocardial infarction. J. Am. Coll. Cardiol., Vol.43, No.12, (Jun, 2004), pp. 2253-2259, ISBN0735-1097

Porto, I., Burzotta, F., Brancati, M., Trani, C., Lombardo, A., Romagnoli, E., Niccoli, G., Natale, L., Bonomo, L. \& Crea, F. (2007) Relation of myocardial blush grade to microvascular perfusion and myocardial infarct size after primary or rescue percutaneous coronary intervention. Am. J. Cardiol., Vol.99, No.12, (Jun, 2007), pp. 1671-1673, ISBN0002-9149

Lund, G.K., Stork, A., Saeed, M., Bansmann, M.P., Gerken, J.H., Müller, V., Mester, J., Higgins, C.B., Adam, G. \& Meinertz, T. (2004) Acute myocardial infarction: evaluation with first-pass enhancement and delayed enhancement $\mathrm{mr}$ imaging compared with 201tl spect imaging. Radiology, Vol.232, No.1, (Jul, 2004), pp. 49-57, ISBN0033-8419

Hombach, V., Grebe, O., Merkle, N., Waldenmaier, S., Höher, M., Kochs, M., Wöhrle, J. \& Kestler, H.A. (2005) Sequelae of acute myocardial infarction regarding cardiac structure and function and their prognostic significance as assessed by magnetic resonance imaging. Eur. Heart J., Vol.26, No.6, (Mar, 2005), pp. 549-557, ISBN0195$668 X$

Habis, M., Capderou, A., Ghostine, S., Daoud, B., Caussin, C., Riou, J., Brenot, P., Angel, C.Y., Lancelin, B. \& Paul, J. (2007) Acute myocardial infarction early viability assessment by 64-slice computed tomography immediately after coronary angiography: comparison with low-dose dobutamine echocardiography. J. Am. Coll. Cardiol., Vol.49, No.11, (Mar, 2007), pp. 1178-1185, ISBN1558-3597

Ito, H., Tomooka, T., Sakai, N., Yu, H., Higashino, Y., Fujii, K., Masuyama, T., Kitabatake, A. \& Minamino, T. (1992) Lack of myocardial perfusion immediately after successful 
thrombolysis. a predictor of poor recovery of left ventricular function in anterior myocardial infarction. Circulation, Vol.85, No.5, (May, 1992), pp. 1699-1705, ISBN0009-7322

Porter, T.R., Li, S., Oster, R. \& Deligonul, U. (1998) The clinical implications of no reflow demonstrated with intravenous perfluorocarbon containing microbubbles following restoration of thrombolysis in myocardial infarction (TIMI) 3 flow in patients with acute myocardial infarction. Am. J. Cardiol., Vol.82, No.10, (Nov, 1998), pp. 1173-1177, ISBN0002-9149

Balcells, E., Powers, E.R., Lepper, W., Belcik, T., Wei, K., Ragosta, M., Samady, H. \& Lindner, J.R. (2003) Detection of myocardial viability by contrast echocardiography in acute infarction predicts recovery of resting function and contractile reserve. J. Am. Coll. Cardiol., Vol.41, No.5, (Mar, 2003), pp. 827-833, ISBN0735-1097

Dwivedi, G., Janardhanan, R., Hayat, S.A., Swinburn, J.M. \& Senior, R. (2007) Prognostic value of myocardial viability detected by myocardial contrast echocardiography early after acute myocardial infarction. J. Am. Coll. Cardiol., Vol.50, No.4, (Jul, 2007), pp. 327-334, ISBN1558-3597

Galiuto, L., Garramone, B., Scarà, A., Rebuzzi, A.G., Crea, F., La Torre, G., Funaro, S., Madonna, M., Fedele, F., Agati, L. (2008) The extent of microvascular damage during myocardial contrast echocardiography is superior to other known indexes of post-infarct reperfusion in predicting left ventricular remodeling: results of the multicenter amici study. J. Am. Coll. Cardiol., Vol.51, No.5, (Feb, 2008), pp. 552-559, ISBN1558-3597

Hashimoto, I., Li, X., Hejmadi Bhat, A., Jones, M., Zetts, A.D. \& Sahn, D.J. (2003) Myocardial strain rate is a superior method for evaluation of left ventricular subendocardial function compared with tissue doppler imaging. J. Am. Coll. Cardiol., Vol.42, No.9, (Nov, 2003), pp. 1574-1583, ISBN0735-1097

Garot, J., Bluemke, D.A., Osman, N.F., Rochitte, C.E., Zerhouni, E.A., Prince, J.L. \& Lima, J.A. (2000) Transmural contractile reserve after reperfused myocardial infarction in dogs. J. Am. Coll. Cardiol., Vol.36, No.7, (Dec, 2000), pp. 2339-2346, ISBN0735-1097

Grattan, M.T., Hanley, F.L., Stevens, M.B. \& Hoffman, J.I. (1986) Transmural coronary flow reserve patterns in dogs. Am. J. Physiol., Vol.250, No.2 Pt 2, (Feb, 1986), p. H276-83, ISBN0002-9513

Corsi, C., Lang, R.M., Veronesi, F., Weinert, L., Caiani, E.G., MacEneaney, P., Lamberti, C. \& Mor-Avi, V. (2005) Volumetric quantification of global and regional left ventricular function from real-time three-dimensional echocardiographic images. Circulation, Vol.112, No.8, (Aug, 2005), pp. 1161-1170, ISBN1524-4539

Inoue, K., Ito, H., Iwakura, K., Kawano, S., Okamura, A., Kurotobi, T., Date, M., Otsu, K., Hori, M. \& Fujii, K. (2006) Usefulness of high-resolution real-time threedimensional echocardiography to visualize the left ventricular endocardial surface in myocardial infarction. Am. J. Cardiol., Vol.97, No.11, (Jun, 2006), pp. 1578-1581, ISBN0002-9149

Iwakura, K., Ito, H., Okamura, A., Kurotobi, T., Koyama, Y., Date, M., Inoue, K., Nagai, H., Imai, M., Arita, Y., Toyoshima, Y., Ozawa, M. \& Fujii, K. (2007) Comparison of twoversus three-dimensional myocardial contrast echocardiography for assessing subendocardial perfusion abnormality after percutaneous coronary intervention in 
patients with acute myocardial infarction. Am. J. Cardiol., Vol.100, No.10, (Nov, 2007), pp. 1502-1510, ISBN0002-9149

Gibson, C.M., Cannon, C.P., Murphy, S.A., Ryan, K.A., Mesley, R., Marble, S.J., McCabe, C.H., Van De Werf, F. \& Braunwald, E. (2000) Relationship of timi myocardial perfusion grade to mortality after administration of thrombolytic drugs. Circulation, Vol.101, No.2, (Jan, 2000), pp. 125-130, ISBN1524-4539

Iwakura, K (2011) . Visualization of Myocardial Hemorrhage with Real Time ThreeDimensional Myocardial Contrast Echocardiography in Patients with Acute Myocardial Infarction. J Echocardiogr, in press

[Cerqueira2002] Standardized myocardial segmentation and nomenclature for tomographic imaging of the heart: a statement for healthcare professionals from the cardiac imaging committee of the council on clinical cardiology of the american heart association. . 2002.

Ragosta, M., Camarano, G., Kaul, S., Powers, E.R., Sarembock, I.J. \& Gimple, L.W. (1994) Microvascular integrity indicates myocellular viability in patients with recent myocardial infarction. new insights using myocardial contrast echocardiography. Circulation, Vol.89, No.6, (Jun, 1994), pp. 2562-2569, ISBN0009-7322

Kapetanakis, S., Kearney, M.T., Siva, A., Gall, N., Cooklin, M. \& Monaghan, M.J. (2005) Realtime three-dimensional echocardiography: a novel technique to quantify global left ventricular mechanical dyssynchrony. Circulation, Vol.112, No.7, (Aug, 2005), pp. 992-1000, ISBN1524-4539

Galiuto, L. \& Iliceto, S. (1998) Myocardial contrast echocardiography in the evaluation of viable myocardium after acute myocardial infarction. Am. J. Cardiol., Vol.81, No.12A, (Jun, 1998), p. 29G-32G, ISBN0002-9149

Costantini, C.O., Stone, G.W., Mehran, R., Aymong, E., Grines, C.L., Cox, D.A., Stuckey, T., Turco, M., Gersh, B.J., Tcheng, J.E., Garcia, E., Griffin, J.J., Guagliumi, G., Leon, M.B. \& Lansky, A.J. (2004) Frequency, correlates, and clinical implications of myocardial perfusion after primary angioplasty and stenting, with and without glycoprotein iib/iiia inhibition, in acute myocardial infarction. J. Am. Coll. Cardiol., Vol.44, No.2, (Jul, 2004), pp. 305-312, ISBN0735-1097

Bolognese, L., Antoniucci, D., Rovai, D., Buonamici, P., Cerisano, G., Santoro, G.M., Marini, C., L'Abbate, A. \& Fazzini, P.F. (1996) Myocardial contrast echocardiography versus dobutamine echocardiography for predicting functional recovery after acute myocardial infarction treated with primary coronary angioplasty. J. Am. Coll. Cardiol., Vol.28, No.7, (Dec, 1996), pp. 1677-1683, ISBN0735-1097

Kaul, S., Jayaweera, A.R., Glasheen, W.P., Villanueva, F.S., Gutgesell, H.P. \& Spotnitz, W.D. (1992) Myocardial contrast echocardiography and the transmural distribution of flow: a critical appraisal during myocardial ischemia not associated with infarction. J. Am. Coll. Cardiol., Vol.20, No.4, (Oct, 1992), pp. 10051016, ISBN0735-1097

Jenkins, C., Moir, S., Chan, J., Rakhit, D., Haluska, B. \& Marwick, T.H. (2009) Left ventricular volume measurement with echocardiography: a comparison of left ventricular opacification, three-dimensional echocardiography, or both with magnetic resonance imaging. Eur. Heart J., Vol.30, No.1, (Jan, 2009), pp. 98-106, ISBN15229645 
Shishido, T., Beppu, S., Matsuda, H., Yutani, C. \& Miyatake, K. (1997) Extension of hemorrhage after reperfusion of occluded coronary artery: contrast echocardiographic assessment in dogs. J. Am. Coll. Cardiol., Vol.30, No.2, (Aug, 1997), pp. 585-591, ISBN0735-1097

Wei, K., Jayaweera, A.R., Firoozan, S., Linka, A., Skyba, D.M. \& Kaul, S. (1998) Quantification of myocardial blood flow with ultrasound-induced destruction of microbubbles administered as a constant venous infusion. Circulation, Vol.97, No.5, (Feb, 1998), pp. 473-483, ISBN0009-7322 


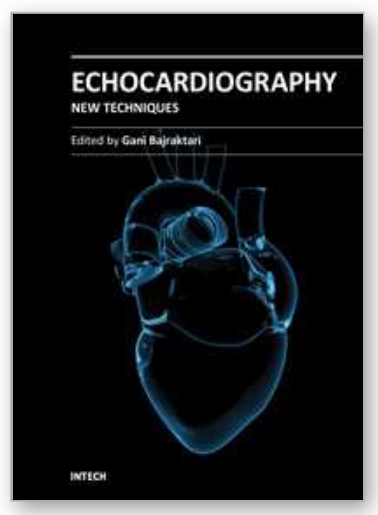

\author{
Echocardiography - New Techniques \\ Edited by Prof. Gani Bajraktari
}

ISBN 978-953-307-762-8

Hard cover, 218 pages

Publisher InTech

Published online 18, January, 2012

Published in print edition January, 2012

The book "Echocardiography - New Techniques" brings worldwide contributions from highly acclaimed clinical and imaging science investigators, and representatives from academic medical centers. Each chapter is designed and written to be accessible to those with a basic knowledge of echocardiography. Additionally, the chapters are meant to be stimulating and educational to the experts and investigators in the field of echocardiography. This book is aimed primarily at cardiology fellows on their basic echocardiography rotation, fellows in general internal medicine, radiology and emergency medicine, and experts in the arena of echocardiography. Over the last few decades, the rate of technological advancements has developed dramatically, resulting in new techniques and improved echocardiographic imaging. The authors of this book focused on presenting the most advanced techniques useful in today's research and in daily clinical practice. These advanced techniques are utilized in the detection of different cardiac pathologies in patients, in contributing to their clinical decision, as well as follow-up and outcome predictions. In addition to the advanced techniques covered, this book expounds upon several special pathologies with respect to the functions of echocardiography.

\title{
How to reference
}

In order to correctly reference this scholarly work, feel free to copy and paste the following:

Katsuomi Iwakura (2012). 3D Myocardial Contrast Echocardiography, Echocardiography - New Techniques, Prof. Gani Bajraktari (Ed.), ISBN: 978-953-307-762-8, InTech, Available from:

http://www.intechopen.com/books/echocardiography-new-techniques/3d-myocardial-contrastechocardiography

\section{INTECH}

open science | open minds

\section{InTech Europe}

University Campus STeP Ri

Slavka Krautzeka 83/A

51000 Rijeka, Croatia

Phone: +385 (51) 770447

Fax: +385 (51) 686166

www.intechopen.com

\section{InTech China}

Unit 405, Office Block, Hotel Equatorial Shanghai

No.65, Yan An Road (West), Shanghai, 200040, China

中国上海市延安西路 65 号上海国际贵都大饭店办公楼 405 单元

Phone: +86-21-62489820

Fax: +86-21-62489821 
(C) 2012 The Author(s). Licensee IntechOpen. This is an open access article distributed under the terms of the Creative Commons Attribution 3.0 License, which permits unrestricted use, distribution, and reproduction in any medium, provided the original work is properly cited. 